\title{
Modal Properties of Two-Dimensional Antiguided Vertical-Cavity Surface-Emitting Laser Arrays
}

\author{
Delai Zhou, Member, IEEE, L. J. Mawst, Senior Member, IEEE, and Zheng Dai, Member, IEEE
}

\begin{abstract}
We present a detailed analysis on 2 -D $(4 \times 4$ square-lattice) antiguided vertical-cavity surface-emitting laser (VCSEL) arrays based on the effective-index model. The calculation shows that the array can operate under 2-D resonant coupling, provided that the resonance condition in both the horizontal and vertical directions is satisfied. Consequently, the resonant-mode edge radiation loss is inversely proportional to the number of array elements along one direction for a $N \times N$ array. Low-edge-loss out-of-phase and adjacent array modes are found to compete with the in-phase resonant mode. While the 3-D gain overlap is not a significant factor in modal discrimination, the introduction of inter-element loss allows the in-phase mode to exhibit the lowest threshold gain for a wide range of inter-element width, $s(\Delta s \approx 0.5 \mu \mathrm{m}$ for $980-\mathrm{nm}$ wavelength devices). The 2-D antiguided array results from shifting the cavity resonance between the element and inter-element regions and is fabricated by selective chemical etching and two-step metalorganic chemical vapor deposition (MOCVD) growth. Diffraction-limited in-phase and out-of-phase array mode operation is observed from top-emitting arrays, depending on the inter-element width. Substrate-emitting array structures are investigated as a means to lower heating and increase the coherent output power.
\end{abstract}

Index Terms-Antiguide, in-phase mode, leaky waves, out-ofphase mode, resonance, semiconductor laser arrays, thermal effects, vertical-cavity surface-emmitting laser (VCSEL).

\section{INTRODUCTION}

$\mathbf{C}$ OHERENT emission from the surface makes vertical-cavity surface-emitting lasers (VCSEL) very attractive light sources for many applications such as printing, telecommunications and data storage. Due to the short gain length and small emitting aperture, single-mode emission has been limited to $5-10 \mathrm{~mW}$ from single-element monolithic VCSELs [1], [2]. As an efficient means to increase the coherent output power, phase-locked VCSEL arrays have been studied extensively for the last few years. The coupled-lasing modes of the VCSEL array represent the allowed bands of propagating Bloch waves in the 2-D active photonic lattice. Previous VCSEL array designs have attempted to select a single-mode (i.e., all elements phase locked) from the allowed bands of guided-modes supported by the 2-D photonic lattice. The conventional approaches start with a large-area VCSEL structure, and incorporate a means to separate the individual array elements [3]-[6] such as: 1) pattern the array reflectivity so that the elements under the higher reflectivity regions have a lower

Manuscript received October 2, 2001; revised February 28, 2002. This work was supported by the National Science Foundation under Grant 9734283.

D. Zhou and L. J. Mawst are with the University of Wisconsin-Madison, Madison, WI 53706 USA (e-mail: mawst@engr.wisc.edu).

Z. Dai is with AlfaLight Inc., Madison, WI 53704 USA.

Publisher Item Identifier S 0018-9197(02)05031-5. threshold gain [3], [4]; 2) restrict the gain (injection current) to individual elements by selective ion implantation [5]; and 3) selectively etch the top surface so that the unetched regions form the higher-cavity- $Q$ array elements [6]. These attempts to fabricate VCSEL arrays have successfully demonstrated phase locking among the array elements, emitting partially-coherent beams. However, stable, high-power, diffraction-limited beam operation from 2-D VCSEL arrays has not been realized. In addition, all previously reported phase-locked 2-D VCSEL arrays operate in either the out-of-phase array mode or a mixture of various array modes. This behavior is characteristic of weakly-index guided or gain-guided phase-locked arrays in which the highest-order (i.e., out-of-phase mode) is always favored to lase over the in-phase mode [7]-[9], [41], [42], with poor intermodal discrimination and mode stability. By changing the phase relationships of adjacent array-elements, such as patterning the 2-D array surface with an external phase-shifter [10], "in-phase mode-like" emission was obtained from optically pumped VCSEL arrays, although the beam is quite broad (twice the diffraction-limit), indicating multi-mode operation. The progress in phase-locked 2-D VCSEL arrays parallels closely that of edge-emitting phase-locked arrays. Weak coupling and poor inter-modal discrimination found in evanescently coupled edge-emitting laser arrays has severely limited their single-mode output power [11].

According to the coupled-mode theory employed to calculate the modal behavior in weakly index-guided array systems, each element only couples with its nearest neighbors by their weak evanescent waves (series-coupling), and each array mode is a superposition of individual element modes [8], [41], [9], [42]. For an $N \times N$ 2-D VCSEL array, the coupled modes can be labeled by the number of intensity nulls in each direction. Using this nomenclature, mode $(1,1)$ is the fundamental in-phase mode, and mode $(N, N)$ is the highest-order out-of-phase mode [3], [12]. Results from 1-D edge-emitting laser arrays [7], [8], [41], [9], [42] indicate that mode $(N, N)$ is always favored to lase over other modes (simply because of a larger gain overlap), and modal discrimination is relatively small. Similarly, 2-D index guided VCSEL arrays also are found to operate in the highest order mode $(N, N)$ or a mixture of high-order modes [3]-[6], [12]. By contrast, antiguided array structures exhibit strong leaky-wave coupling (parallel coupling) with array-mode dependent lateral radiation loss, leading to high inter-modal discrimination [13]. As a result, edge-emitting antiguided arrays have demonstrated coherent output power in the watt-range [14]. The large built-in index step $(>0.02)$ and strong lateral radiation leakage from an antiguide make them well suited for array integration. Moreover, the strong 
leaky-wave coupling enables the array structure with relatively large element spacing. This may be more beneficial for the 2-D array case due to the more severe heating problem. In contrast with the positive-index guided VCSEL arrays, the antiguided VCSEL array operates in the allowed leaky-mode bands of the 2-D photonic lattice.

Antiguided VCSELs have been demonstrated by either the regrowth of high-index material in a buried heterostructure design [15], selective oxidation [16] or by a cavity-induced resonant shifted structure [17]. In 1999, Serkland et al. [18] demonstrated leaky-wave coupling between two antiguided VCSELs (coupled in-phase or out-of-phase) using structures fabricated by selectively etching down the cavity to generate an antiguiding effect. In 2000, we reported the first diffraction-limited (in-phase mode) on-axis emission from a monolithic $4 \times 4$ $6 \mu \mathrm{m}$-square-element antiguided VCSEL array [19]. Moreover, the emission pattern (either in-phase or out-of-phase mode operation) can be totally controlled by the 2-D array structure, such as by adjusting the array spacing or built-in index step [18], [19]. In this paper, we conduct a comprehensive cold-cavity study of the 2-D antiguided (square-geometry) array modal behavior using the effective-index model and fiber-mode approximation method [20]. Calculations show that resonant coupling [21]-[23] for the in-phase (out-of-phase) mode occurs when the inter-element spacing is close to an odd (even) integral number of half-waves of the antiguide radiation leakage, and that the use of inter-element loss together with the mode-dependent radiation loss can be effective in suppressing nonresonant modes. In addition, other array-geometries such as triangular-lattice (6-nearest neighbors) can also exhibit lateral resonant coupling, providing a broad design space for optimization. CW output power from previously reported top-emitting structures was limited to $1-2 \mathrm{~mW}$, partly due to the intensive heating resulting in output power saturation. Thus, a bottom-emitting design is essential to improve the efficiency and output power of large-number 2-D VCSEL arrays [24].

\section{2-D ANTIGUided VCSEL ARRAY STRUCTURE: EFFECTIVE-INDEX MODEL}

Due to the difficulties in studying a 3-D multilayer structure, we utilize the effective-index model and fiber-mode approximation to calculate the modal behavior of the VCSEL array structure [20]. In this approximation, the array elements and outer regions are simply represented by the effective indices $n_{0}$ and $n_{1}$, respectively, calculated from the 1-D transverse layered structure in each region. The 3-D structure is then transferred into a 2-D "fiber array," as shown in Fig. 1. According to the effective-index model for a VCSEL proposed by Hadley [25], a red shift of the resonant wavelength corresponds to an increase of effective index, given by

$$
\Delta n / n_{0}=\Delta \lambda / \lambda_{0}
$$

where $n_{0}$ and $\lambda_{0}$ are the effective index and resonant wavelength, respectively, in the array element region.

One reason we apply this method here is that the array structure is only in the lateral direction; thus, we are interested in the

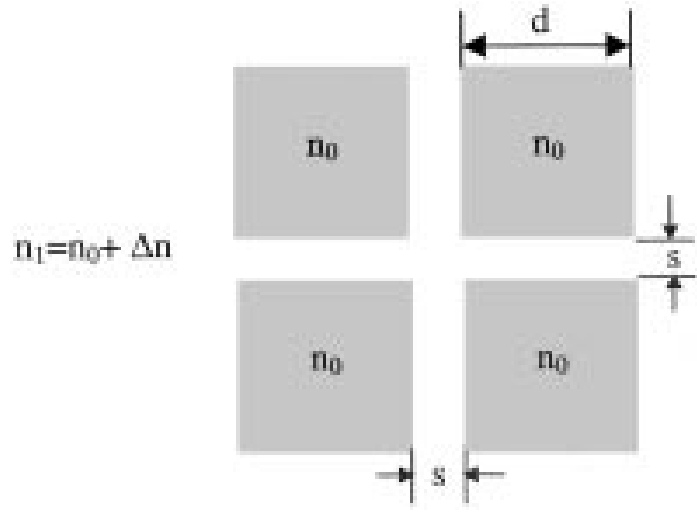

Fig. 1. Effective-index modeling structure for a $2 \times 2$ array with $\Delta n=0.05$ and $d=6 \mu \mathrm{m}: s$ represents the inter-element spacing and $n_{0}$ is the effective index of the element region.

modal behavior in this direction. The "fiber-modes" can readily be solved by various numerical solutions of the scalar Helmholtz equation, such as finite-difference calculations with the perfect matched layer (PML) boundary conditions. For simplicity, we studied the leaky mode behavior of a $4 \times 4$ antiguided VCSEL array structure as shown in Fig. 1 (only a truncated $2 \times 2$ version of the $4 \times 4$ array is shown). The 16-square regions represent the array elements that have effective-indices $\left(n_{0}\right)$ lower than that $\left(n_{1}\right)$ in the inter-element and background regions. Similar to the 1-D antiguided array case [21], [22], these 2-D structures support leaky modes with the elements phase locked either in-phase or out-of-phase, as well as intermediate modes with varying phase relationships between elements. The leaky modes have mode propagation constants which lie below the lowest effective index of the structure and exhibit mode-dependent edge radiation losses. From the point of view of a simple summation, we expect the optical coupling and modal discrimination of 2-D array structures to be stronger than in the 1-D array case.

Consider a single antiguide (Fig. 2) with a core index of $n_{0}$ and index step of $\Delta n=n_{1}-n_{0}$. The lowest loss leaky mode (fundamental antiguided mode) exhibits a radiating field into the high-index cladding regions characterized by a lateral wavelength $\lambda_{1}$. The lateral wavelength $\lambda_{1}$ of the radiation leakage can be approximated by [26]

$$
\lambda_{1}=\lambda_{0} /\left(n_{1}^{2}-n_{0}^{2}+\lambda_{0}^{2} / 4 d^{2}\right)^{1 / 2}
$$

where $\lambda_{0}$ is the vacuum wavelength and $d$ is the low-index "core" width.

For a 1-D antiguided array, each element leaks radiation into neighboring elements, with a lateral wavelength $\lambda_{1}$ also approximated by (2). When the array inter-element spacing, $s$, satisfies the resonant condition

$$
\begin{aligned}
s & =m \lambda_{1} / 2 \\
\text { in phase mode resonance, } \quad m & =\text { odd number } \\
\text { out-of-phase mode resonance, } m & =\text { even number }
\end{aligned}
$$

and the array exhibits resonant coupling due to the lateral radiation leakage. As a result, the resonant mode (either in-phase or out-of-phase) has a uniform near-field profile across the array and negligible inter-element field. At resonance, each leaky wave has maximum transmission passing through the 


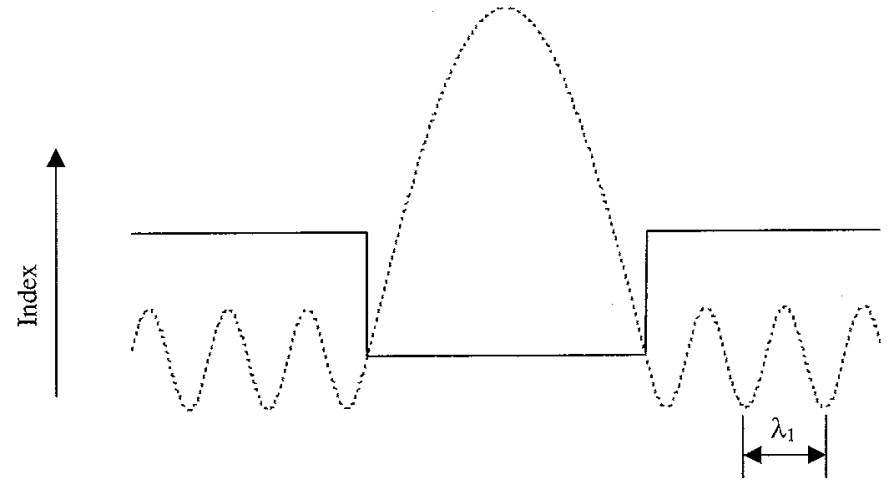

Fig. 2. 1-D structure of a single antiguide: the dotted curve is the calculated optical field for the fundamental leaky mode.

inter-element region and couples uniformly with other elements, causing very little light to be trapped in the inter-element region. The leaky array modes can easily be calculated by the means of a transfer matrix method [27], and are plotted in Fig. 3 for selected modes. For a fixed index-step $(\Delta n=0.05)$, Fig. 3(a)-(c) shows the resonant out-of-phase $(s=1.7 \mu \mathrm{m})$, resonant in-phase $(s=2.5 \mu \mathrm{m})$ and its adjacent mode profiles, respectively. It has been demonstrated both theoretically [28] and experimentally [14] that the resonant (or near resonant) mode with a uniform near field is relatively insensitive to gain spatial hole burning and has the potential for high-power single-mode operation. In order to select the resonant in-phase mode that has an on-axis beam pattern, several mode selection mechanisms have been studied in 1-D antiguided arrays, such as inter-element loss [29], 2-D gain overlap factor [30], and the Talbot-type spatial filter [31], [32]. While the spatial filter is obviously not suited for the monolithic VCSEL array, the first two mechanisms are built-in to the antiguide structure and their role in mode selection for the 2-D VCSEL array needs to be evaluated.

The number of standing wave nulls determines the mode identification notation for a 1-D array with $N$ elements. That is, the mode $L$ can be defined as (assume each element contains a fundamental mode)

$$
L=(m+1) *(N-1)
$$

where $m$ is the number of standing wave peak in the inter-element region [26]. For example, the mode in Fig. 3(a) represents mode 9, and the one in Fig. 3(b) represents mode 12.

It is also logical to define the 2-D $N \times N$ array's leaky modes as a superposition of two 1-D array modes. As we will show later, we find that the leaky-wave coupling in the two directions is independent. This means that, if in one direction the leaky mode number is $m$ and the other direction is mode $n$, then we can define this 2-D array mode as mode $(m, n)$. The details will be described in the following parts.

\section{Modal Behavior (Without Inter-Element Loss)}

\section{A. Mode Profiles}

The 2-D in-phase and out-of-phase lateral modes are calculated using the effective-index approximation for an array with element width $d$ of $6 \mu \mathrm{m}$ and index step $\Delta n$, of $0.05\left(n_{0}=\right.$

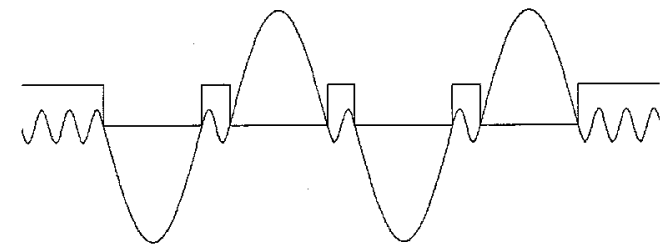

(a)

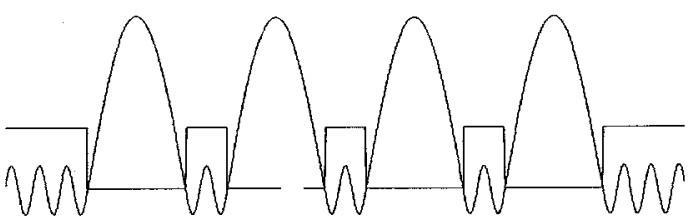

(b)

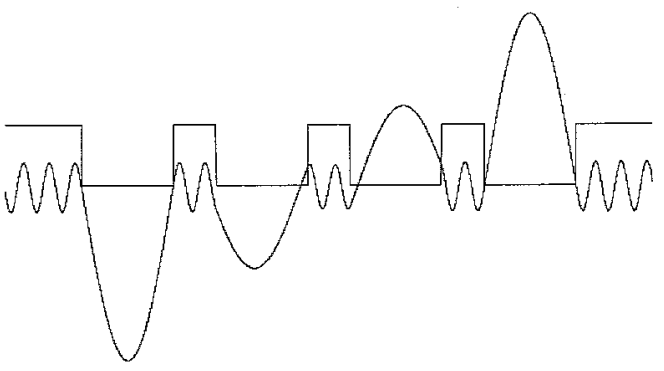

(c)

Fig. 3. Calculated field profiles of a 1-D four-element antiguided array. (a) Resonant out-of-phase mode. (b) Resonant in-phase mode. (c) Adjacent mode.

$\left.3.3, n_{1}=3.35\right)$. From (2), the lateral wavelength of the radiation leakage from each element is

$$
\lambda_{1}=1.68 \mu \mathrm{m} .
$$

For a 1-D array with this design, the out-of-phase and in-phase mode resonance occurs for interelement width $s=\lambda_{1}$ (1.68 $\mu \mathrm{m})$ and $1.5 \lambda_{1}(2.5 \mu \mathrm{m})$ respectively. While we expect similar values for $s$ at resonance with the 2-D structure, there may be some differences as a result of the 2-D geometry.

In order to examine the optical coupling behavior among array elements, we follow the development of the out-of-phase mode $(9,9)$ and the in-phase-mode $(12,12)$ while varying the inter-element distance, $s$, as shown in Fig. 4. Along each axis of the 2-D mode field profile, the number of lobes between two nearest square element regions corresponds to the number of standing wave peaks as in the 1-D case which was shown in Fig. 3. For example, there are always three peaks for mode $(12,12)$ and two peaks for mode $(9,9)$ between any two nearest squares of the 2-D array. For a 1-D array, either the in-phase mode or the out-phase-mode will be cut off if $s$ is not within a certain range of resonance, such as a half-lateral wavelength away from its resonance position [26]. Although there are no analytical solutions for the more complicated 2-D array geometry, the cutoff conditions can be determined from the simulation directly. That is, for the 2-D case, we find a similar situation to the 1-D array: mode $(9,9)$ is cut off when $s$ is larger than $3 / 2 \lambda_{1}$; mode $(12,12)$ exists only between $s$ values of $\lambda_{1}$ and $2 \lambda_{1}$; and mode $(15,15)$ exists only above $s=1.5 \lambda_{1}$. Starting from $s=1.2 \mu \mathrm{m}$, the out-of-phase mode $(9,9)$ has a very nonuniform (cosine shape) near field, characteristic of 


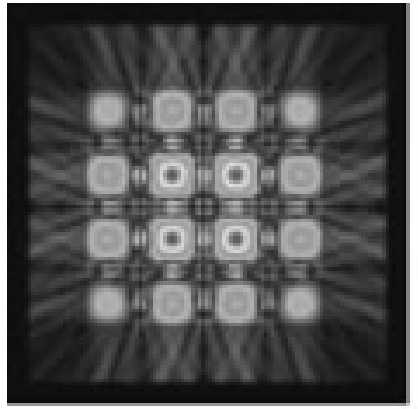

$\mathrm{g}=1.2 \mu \mathrm{m}$

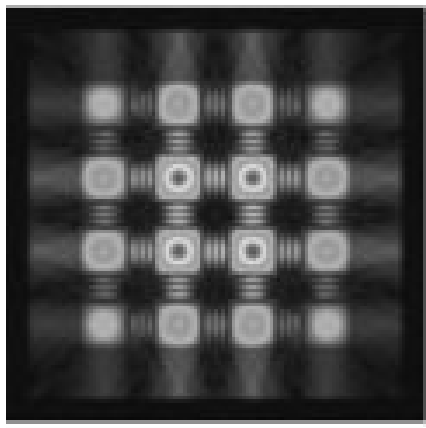

$\mathrm{s}=20 \mathrm{\mu m}$

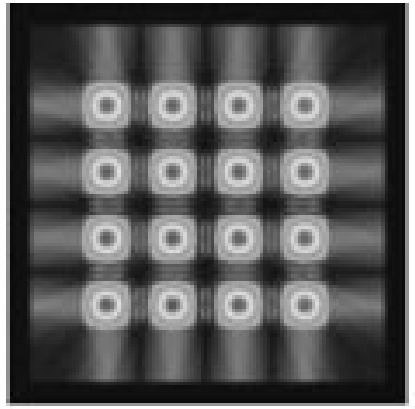

$\mathrm{s}=1.6 \mu \mathrm{m}$

(a)

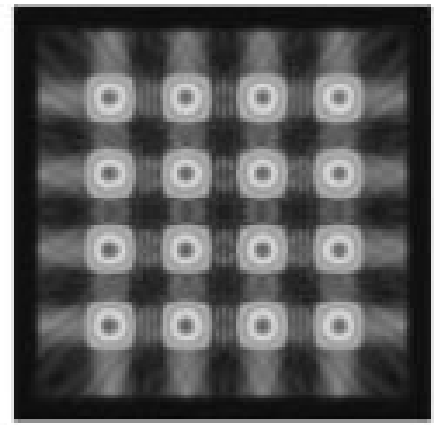

$\mathrm{s}=2.4 \mu \mathrm{m}$

(b)

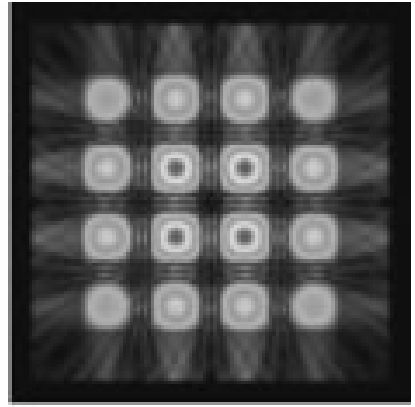

$\S=2.0 \mu \mathrm{m}$

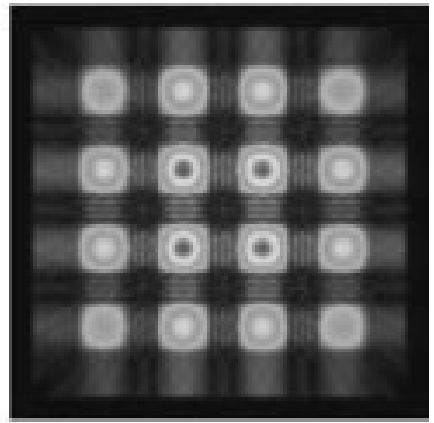

$s=2.8 \mu \mathrm{m}$

Fig. 4. Calculated 2-D field profiles of a $4 \times 4$ antiguided VCSEL array with an index step $\Delta n=0.05$ for various inter-element spacing $s$ for: (a) out-of-phase modes at $s=1.2,1.6$, and $2.0 \mu \mathrm{m}$ and (b) in-phase modes at $s=2.0,2.4$, and $2.8 \mu \mathrm{m}$.

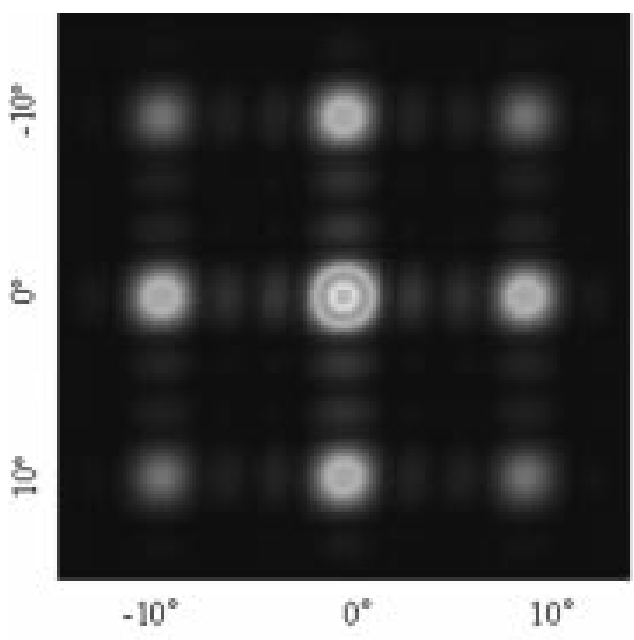

(a)

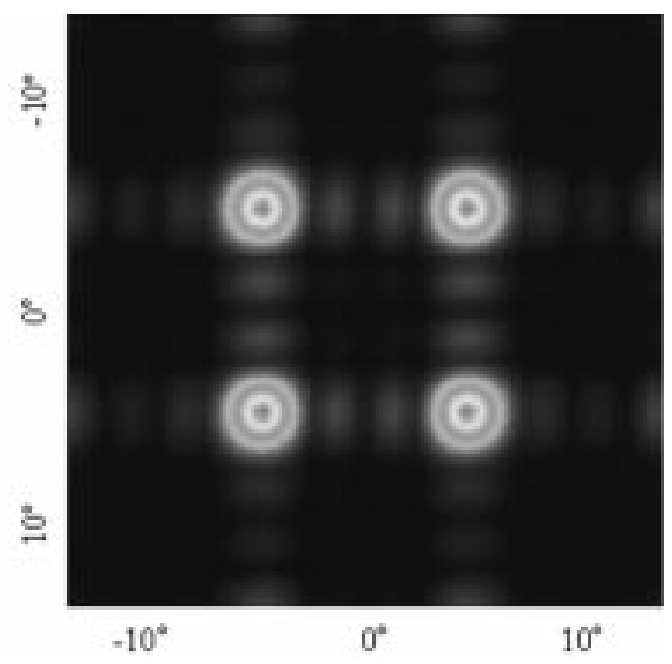

(b)

Fig. 5. Calculated 2-D Far-field patterns for: (a) in-phase mode and (b) out-of-phase mode of a $4 \times 4$ array.

nonresonant modes [Fig. 4(a)]. At $s=1.6 \mu \mathrm{m}\left(\sim \lambda_{1}\right)$, the out-of-phase mode $(9,9)$ exhibits a nearly uniform near-field profile, characteristic of the lateral resonance. As $s$ increases to $2.0 \mu \mathrm{m}$, the field becomes more like a cosine-shaped profile again. The nonuniform field profile of the nonresonant mode is vulnerable to gain-spatial-hole-burning (GSHB) and thermal lensing, leading to multi-mode operation. The reverse process occurs for mode $(12,12)$, as shown in Fig. 4(b). At $s=2.0 \mu \mathrm{m}$, it has a nonuniform near field, as $s$ approaches $2.5 \mu \mathrm{m}\left(1.5 \lambda_{1}\right.$, the in-phase resonance position), and the field profile becomes increasingly uniform. After passing the resonant point, the field becomes nonuniform again. The uniform field profile at resonance leads to a reduced sensitivity to GSHB [28]. This 2-D modal behavior demonstrates that the 2-D resonance occurs while the inter-element spacing in both of the 1-D directions closely satisfies (3).

Fig. 5 shows the calculated far-field patterns for both the in-phase (all elements are in-phase) and out-of-phase (nearest neighbors are out-of-phase) modes. Because of the square symmetry of the lattice, the in-phase mode always has the main 
TABLE I

COMPUTED RAdiation LOSS OF THE RESONANT IN-PHASE MODE FOR $N \times N$ ANTIGUIDED VCSEL ARRAYS WITH $\Delta n=0.05, d=6 \mu \mathrm{m}$, AND $s=2.4 \mu \mathrm{m}$

\begin{tabular}{l|l|l|l}
\hline Array Size & $4 \times 4$ & $2 \times 2$ & $1 \times 1$ \\
\hline Radiation Loss $\left(\mathrm{cm}^{-1}\right)$ & 11.16 & 22.45 & 44.33 \\
\hline
\end{tabular}

power in the center lobe with surrounding smaller lobes forming a square pattern, and the out-of-phase mode has its power divided into 4 primary lobes.

At resonance, the $N$-element array has uniform field-intensity with each element containing a fundamental mode. Therefore, the entire array can be approximated as a single antiguide with width $N * d$ and operating in the $(N-1)$ th-order mode, which has $N$ peaks. Because the radiation loss for the $m$ th-order mode of a single antiguide is proportional to the square of $(m+$ 1) and inversely proportional to $d^{3}$, as given below [33]

$$
\alpha_{1}=(m+1)^{2} \lambda_{0} \lambda_{1} \Gamma^{2} /\left(d^{3} n_{0}\right)
$$

where $\Gamma$ is the lateral confinement factor, the radiation loss of the resonant in-phase or out-of-phase mode of an $N$-element array is roughly proportional to $1 /\left(N d^{3}\right)$. Therefore, for an $N \times N$ array, the resonant mode loss should also be proportional to $1 / N$ provided that the coupling is independent along the vertical and horizontal directions. That is, we expect

$$
\alpha_{N \times N}=\alpha_{1 \times 1} / N
$$

where $\alpha_{N \times N}$ and $\alpha_{1 \times 1}$ are the radiation losses for the $N \times N$ and $1 \times 1$ (a single square antiguide) array, respectively. The computed radiation losses for $1 \times 1,2 \times 2$, and $4 \times 4$ arrays resonant in the in-phase mode are shown in Table I. The nearly linear relationship indicates that (7) is a good approximation for the 2-D array losses. More generally, we expect the radiation loss for a rectangular $M \times N$ 2-D array $\alpha_{M \times N}$ to be

$$
\alpha_{M \times N}=\alpha_{1 \times 1}(1 / M+1 / N) / 2
$$

under the assumption that the coupling in the two directions is independent.

\section{B. Modal Discrimination}

1) In-Phase and Out-of-Phase Modes: Designing the array structure to favor oscillation in the desirable in-phase mode requires an understanding of the intermodal discrimination mechanisms. One mode selection mechanism in the antiguided array is lateral mode-dependent radiation loss. The calculated modal edge radiation loss for selected array modes as a function of interelement spacing, $s$, is shown in Fig. 6. The resonant modes (at $s \sim 1.6,2.5 \mu \mathrm{m}$ ) have maximum loss, while the nonresonant modes have lower losses with the anti-resonant modes $(s \sim$ $2.2,3.0 \mu \mathrm{m}$ ) having the minimum. This can be explained qualitatively as follows. The intensity transmission rate of the lateral leaky wave $\left(\lambda_{1}\right)$ passing through the inter-element region is given by the transmission of a Fabry-Perot etalon [27]

$$
T=(1-R)^{2} /\left[(1-R)^{2}+4 R \sin ^{2} \phi\right]
$$

where $R$ is the intensity reflection at the boundary between element and inter-element regions and the phase-shift value $\phi=$

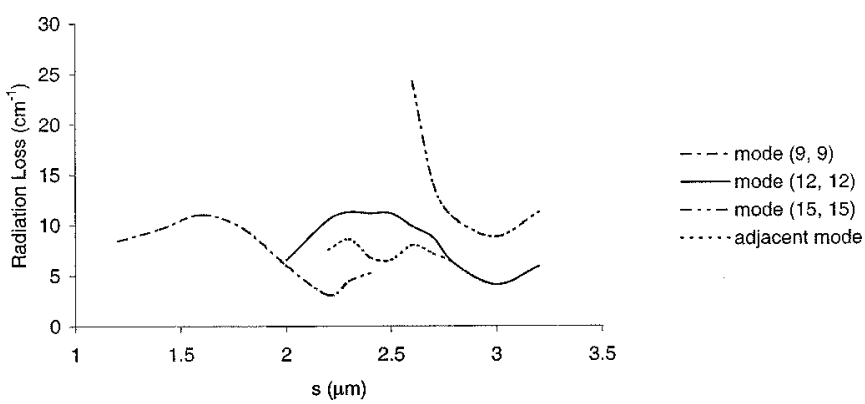

Fig. 6. Calculated radiation losses as a function of inter-element spacing $s$ for in-phase mode $(12,12)$, out-of-phase modes $(9,9),(15,15)$, and relevant adjacent modes discussed in Fig. 2(b).

$2 \pi s / \lambda_{1}$. Under the resonant condition when the inter-element spacing $s$ is equal to an integer times of $\lambda_{1} / 2$, the radiation from each element has maximum transmission through it. While this type of maximum transmission generates maximum coupling among elements (resulting in a uniform near-field), the total radiation is also a maximum and very little field is trapped inside the inter-element region. By contrast, when $s$ is equal to an odd number of quarter waves $\left(\lambda_{1} / 4\right)$ (anti-resonant condition), the radiation undergoes minimum transmission through each inter-element region and thus is trapped inside the high-index interelement regions. Therefore, these anti-resonant modes have weaker coupling (mostly via the trapped leaky waves from the two nearest-neighbor antiguided elements), resulting in a cosine-shaped near-field that is similar to the "coupled mode" behavior. The high field intensity trapped in the inter-element regions leads to a minimum array mode radiation loss.

According to the previous discussion, the cutoff value of the inter-element spacing $s_{m}$ for any in-phase or out-of-phase mode $(m, m)$ can be approximated as

$$
s_{m-3}<s_{m}<s_{m+3}
$$

where $s_{m-3}$ and $s_{m+3}$ are the resonant positions for mode ( $m-$ $3, m-3)$ and mode $(m+3, m+3)$, respectively. From our simulation, as $s$ approaches the cutoff value, the cutoff mode will transform into another mode, such as coupled guided-array modes or coupled higher order leaky modes. The guided-array modes can be eliminated effectively by placing absorptive material within the high-index inter-element regions (as will be discussed later). The coupled higher order leaky modes always have large edge losses that suppress their lasing. We also see from Fig. 6 that the higher order out-of-phase mode $(15,15)$ always has higher edge loss than the in-phase mode $(12,12)$ for $s$ values in the range 2.6-3.0 $\mu \mathrm{m}$. However, for the range of $s$ between $2.0-2.5 \mu \mathrm{m}$, the lower order out-of-phase mode $(9,9)$ will be favored to lase, based on edge radiation losses alone.

2) Adjacent Array Modes: Other than the in-phase and out-of-phase leaky modes mentioned above, there are many intermediate-order modes between them, called adjacent modes. For example, mode $(9,10)$ is an adjacent mode with nine nulls in one direction and 10 nulls in another direction. In a 1-D array, the adjacent modes exhibit larger radiation loss than the in-phase mode on the longer $s$ side of the resonance position [26], because of the large field intensity at the edges of the array [see Fig. 3(c)], and thus can be suppressed. We 


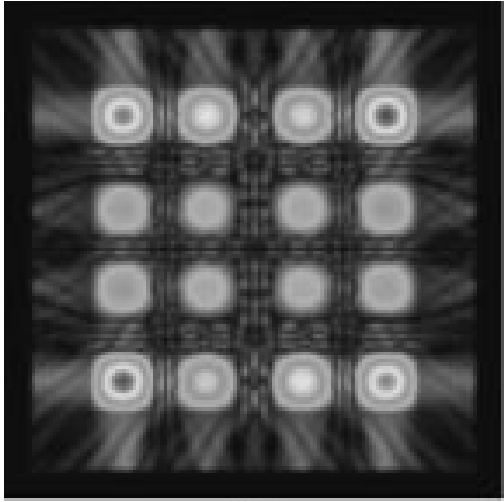

(3)

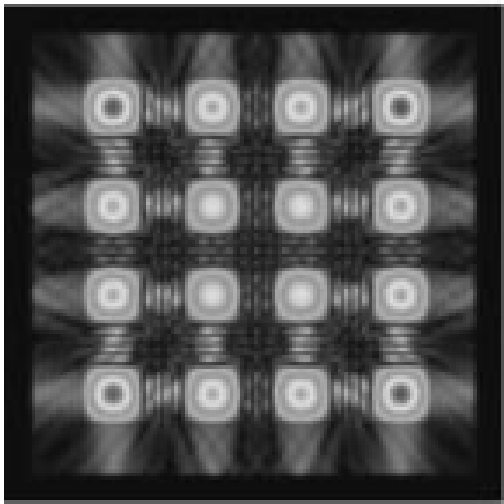

$(\varepsilon)$

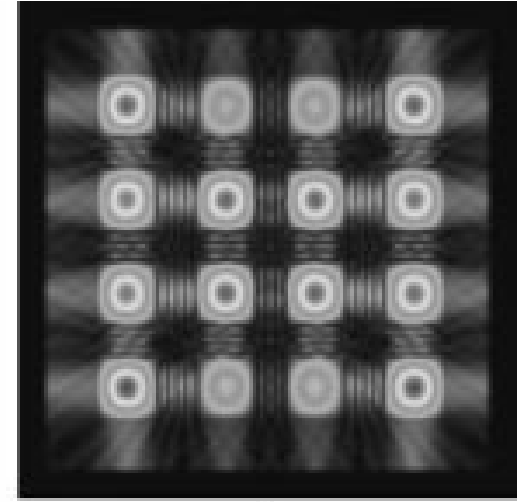

(b)

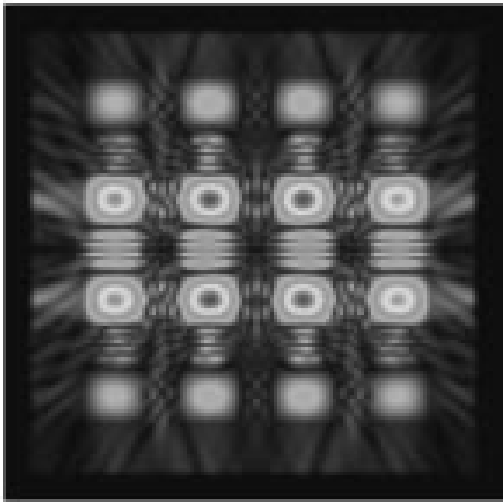

(d)

Fig. 7. Calculated field profiles of adjacent modes with different coupling along the horizontal and vertical directions. (a) Mode (10, 11). (b) Mode (14, 12). (c) Mode (14, 14). (d) Mode (12, 15).

TABLE II

COMPUTED RADIATION LOSS OF THE RELEVANT ADJACENT MODES FOR A $4 \times 4$ ANTIGUIDED VCSEL ARRAY WITH $\Delta n=0.05, d=6 \mu \mathrm{m}$, AND $s=2.4 \mu \mathrm{m}$

\begin{tabular}{l|l|l|l|l|l}
\hline Mode Number & $(9,11)$ & $(9,12)$ & $(10,12)$ & $(11,11)$ & $(12,12)$ \\
\hline Radiation Loss $\left(\mathrm{cm}^{-1}\right)$ & 9.10 & 6.80 & 10.39 & 14.49 & 11.16 \\
\hline
\end{tabular}

find this is not always true of the adjacent modes for the 2-D array structure, due to the 2-D nature of the coupling. For adjacent modes of the 2-D array with high field intensity at the edges of the array, the losses are higher than the loss of the in-phase or out-of-phase modes, similar to the 1-D case [such as mode $(11,10)]$. However, for the 2-D structure, there also exist adjacent modes that have high loss in one direction, but are resonant or antiresonant in the other direction [such as mode $(9,10)]$. We find these adjacent modes have radiation losses comparable to the in-phase or out-of-phase mode. Other adjacent modes [such as mode $(9,12)]$ have in-phase coupling in one direction and out-of-phase coupling in another direction, resulted an even lower radiation loss. Fig. 7 shows several adjacent modes [mode $(10,11),(14,12),(14,14)$ and $(9,12)]$ with different modal properties in the two directions. Table II shows the relevant adjacent mode losses at the in-phase resonant position $(s=2.4 \mu \mathrm{m})$. The calculated edge radiation losses as a function of $s$ with only the most competitive adjacent modes are plotted in Fig. 6. The conclusion is that the in-phase mode
$(12,12)$ is not favored for $s$ between 2.6-3.0 $\mu \mathrm{m}$ (i.e., around the resonant point) due to the competing low-loss adjacent modes. However, the near-resonant in-phase mode possesses the least field in the inter-element regions (refer to Figs. 3 and 4). As we discuss below, this factor allows the 2-D VCSEL array to operate in a resonant or near-resonant in-phase mode, if inter-element losses are introduced into the structure.

\section{RESONANT MODE SELECTION}

\section{A. 3-D Gain Overlap}

In edge-emitting antiguided arrays, the 2-D gain overlap suppresses nonresonant modes, because they exhibit high field intensity in the inter-element regions that have lower transverse optical overlap with the active layer than the field in the element regions have. However, in the antiguided VCSEL array with uniform current injection, the 3-D gain overlap is not expected to be significantly mode-dependent. If we assume a unity lateral field overlap (i.e., 1-D transverse structure), the threshold gain condition for a VCSEL structure with average intrinsic losses $\alpha_{a}$ and $\alpha_{i}$ in the active and passive regions (i.e., distributed Bragg reflector (DBR) and spacer regions), respectively, is [34]

$g_{\mathrm{th}}=\alpha_{a}+\Gamma_{t}^{-1} d^{-1}\left[\alpha_{i}\left(L_{\mathrm{eff}}-d\right)+\alpha_{R} L_{\mathrm{eff}}+\ln \left(1 / R_{1} R_{2}\right)^{1 / 2}\right]$ 


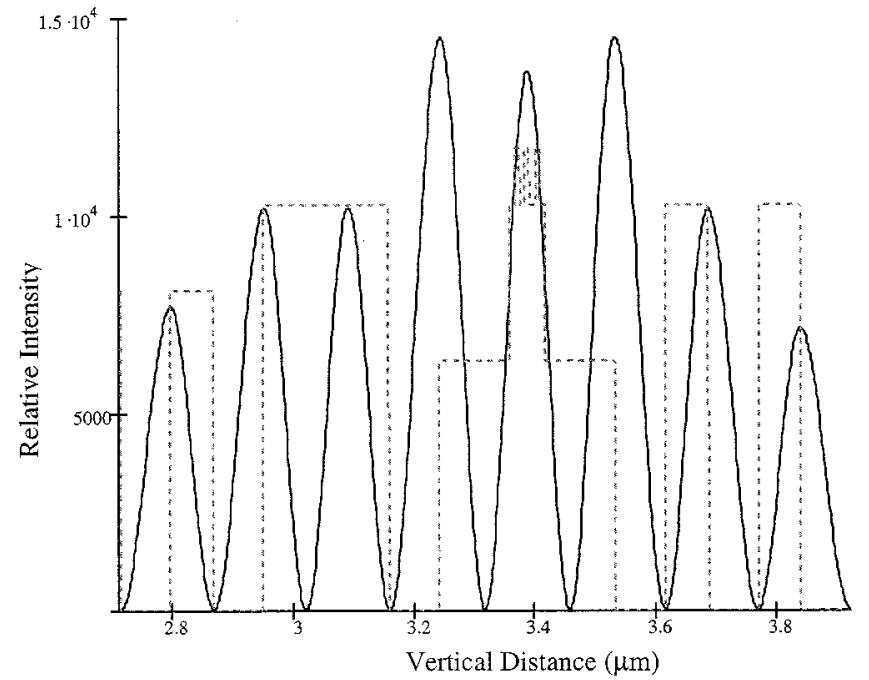

(a)

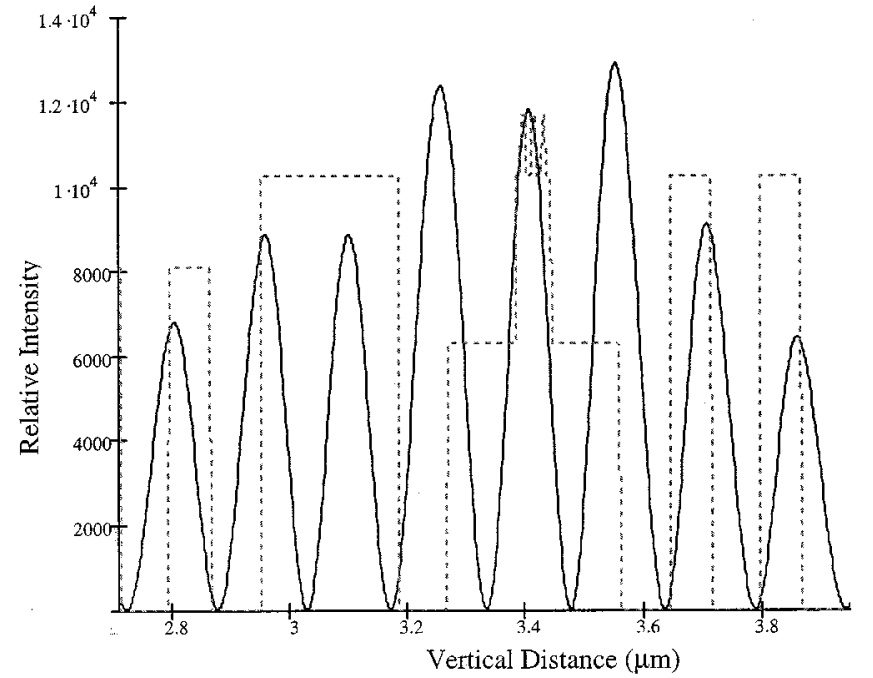

(b)

Fig. 8. Calculated transverse standing wave distribution for: (a) low-index region and (b) high-index region.

where $L_{\text {eff }}$ is the effective cavity length, $d$ is the total active region thickness, $\alpha_{R}$ is the modal radiation loss calculated in Section III, $R_{1}$ and $R_{2}$ are the reflectivity of top and bottom DBR mirrors, respectively, and $\Gamma_{t}$ is the transverse relative confinement factor given by

$$
\Gamma_{t}=\frac{\int_{d}(|E(x)|)^{2} d x}{\int_{L}(|E(x)|)^{2} d x} * \frac{L}{d}
$$

where the integrals are taken over the active and entire cavity regions. If we now include a nonunity mode dependent lateral field overlap, reflecting the fraction of optical field within each VCSEL element of the array, the threshold gain equation should be modified as

$$
\begin{aligned}
\left(g_{\mathrm{th}}-\alpha_{a}\right) & d\left(\Gamma_{l} \Gamma_{e}+\left(1-\Gamma_{l}\right) \Gamma_{i}\right) \\
& =\alpha_{i}\left(L_{\mathrm{eff}}-d\right)+\alpha_{R} L_{\mathrm{eff}}+\ln \left(1 / R_{1} R_{2}\right)^{1 / 2}
\end{aligned}
$$

where $\Gamma_{l}$ is the lateral overlap factor for the field in the element regions, $\Gamma_{e}$ and $\Gamma_{i}$ are the transverse relative confinement factors, defined by (12), in the element and inter-element regions, respectively. It is clear from (13) that, if $\Gamma_{e}$ is larger than $\Gamma_{i}$, the resonant modes (having less field in the inter-element regions, and thus larger $\Gamma_{l}$ ) will have lower threshold gain than the nonresonant modes. From a 1-D VCSEL simulation using the transfer matrix method, $\Gamma_{e}$ is always larger than $\Gamma_{i}$ but the difference is small. This can be seen from the calculated transverse field profiles in the element and interelement regions (Fig. 8). For our antiguided VCSEL array (as described in Section VI) with $\Delta n=0.05, \Gamma_{e}=1.744, \Gamma_{i}=1.724$, $\alpha_{a}=20 \mathrm{~cm}^{-1}, \alpha_{i}=20 \mathrm{~cm}^{-1}, \alpha_{R}=10 \mathrm{~cm}^{-1}, L_{\mathrm{eff}}=1.2 \mu \mathrm{m}$, $R_{1}=99.5 \%, R_{2}=99.9 \%$, and $\Gamma_{l}=0.98$ and 0.92 for the resonant and nonresonant modes, respectively, we obtain from (13) that the threshold gain difference between resonant and nonresonant modes is $\Delta g_{t h} \sim 1 \mathrm{~cm}^{-1}$, which has a negligible effect on the threshold current densities.

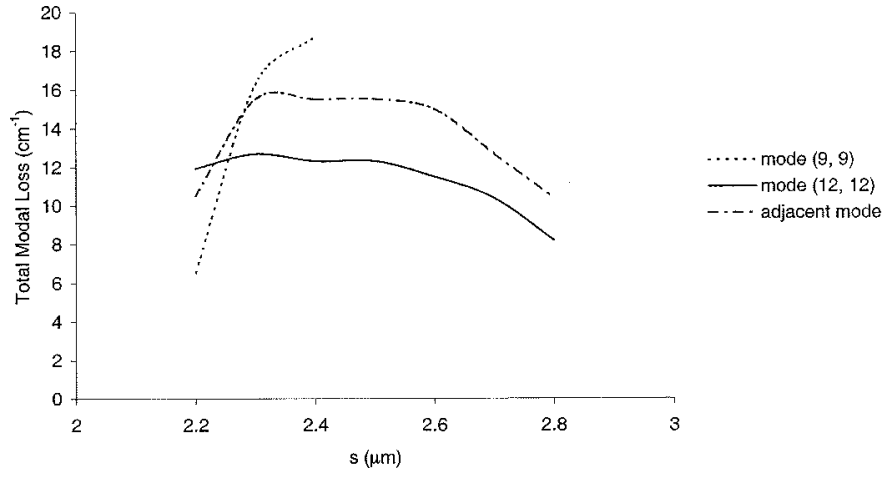

Fig. 9. Calculated modal losses (radiation loss + absorption loss) of competing 2-D array modes for a structure including the addition of interelement loss $\left(100 \mathrm{~cm}^{-1}\right)$. For this structure, the in-phase mode is favored over a large range of interelement spacing $(s)$.

\section{B. Inter-Element Loss}

If the intrinsic loss $\alpha_{i}$ is different between the element and inter-element regions, such as $\alpha_{1}$ in the element region and $\alpha_{2}$ in the inter-element region, (13) can be rewritten as

$$
\begin{aligned}
& \left(g_{\mathrm{th}}-\alpha_{a}\right) d\left[\Gamma_{l} \Gamma_{e}+\left(1-\Gamma_{l}\right) \Gamma_{i}\right] \\
& =\Gamma_{l} \alpha_{1}\left(L_{\mathrm{eff}}-d\right)+\left(1-\Gamma_{l}\right) \alpha_{2}\left(L_{\mathrm{eff}}-d\right) \\
& \quad+\alpha_{R} L_{\mathrm{eff}}+\ln \left(1 / R_{1} / R_{2}\right)^{1 / 2} .
\end{aligned}
$$

In order to suppress the nonresonant modes with larger (1 $\Gamma_{l}$ ), an increase of $\alpha_{2}$ should be beneficial. For example, if we keep $\alpha_{1}=20 \mathrm{~cm}^{-1}$ and increase $\alpha_{2}$ to $120 \mathrm{~cm}^{-1}$, and use the previous parameters, we obtain $g_{\mathrm{th}_{\mathrm{rcsonant}}}=1877 \mathrm{~cm}^{-1}$ and $g_{\mathrm{th}_{\text {nonresonant }}}=2072 \mathrm{~cm}^{-1}$. We can then calculate the threshold current density for the resonant and nonresonant mode: $J=$ $B N_{\mathrm{th}}^{2} q d / \eta_{i}$ and $N_{\mathrm{th}}=N_{\mathrm{tr}} e^{g_{t h} / g_{0}}$. With the assumption that $N_{\mathrm{tr}}=1.8 * 10^{18} \mathrm{~cm}^{-3}, g_{0}=2100 \mathrm{~cm}^{-1}, B=10^{-10}$ $\mathrm{cm}^{3} \mathrm{~s}^{-1}$ and $\eta_{i}=1$, the threshold current density is calculated to be $J_{\text {th }_{\text {rcsonant }}}=651 \mathrm{~A} / \mathrm{cm}^{2}$ and $J_{\mathrm{th}_{\text {nonresonant }}}=783 \mathrm{~A} / \mathrm{cm}^{2}$. By comparison, if we convert the threshold gain from the previous section (no inter-element loss) into $J_{\text {th }}$, we obtain $J_{\text {th }}=$ $613 \mathrm{~A} / \mathrm{cm}^{2}$. The above discussion shows that: 1) the introduction of inter-element loss can effectively suppress the nonreso- 


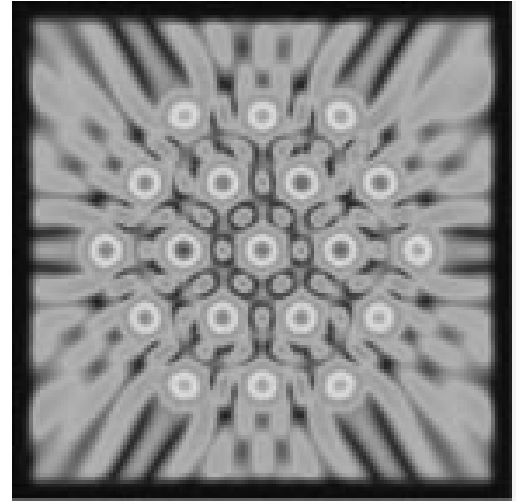

$8 \mu_{\mathrm{m}}$

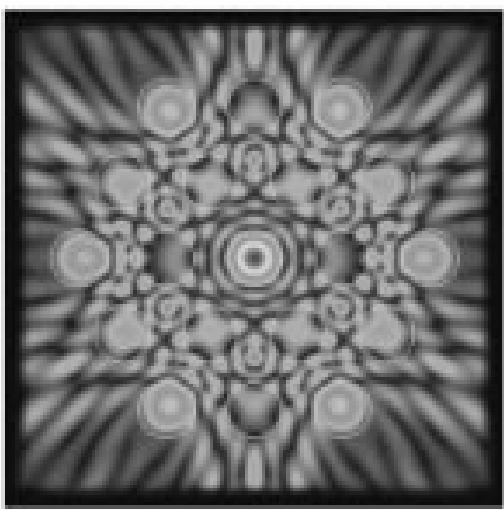

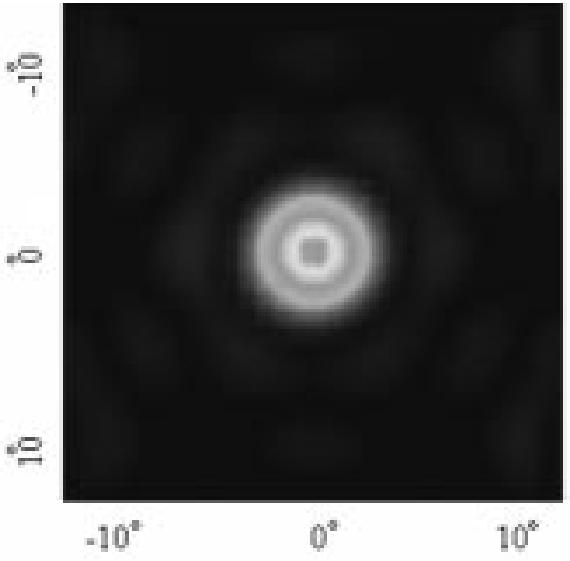

(a)

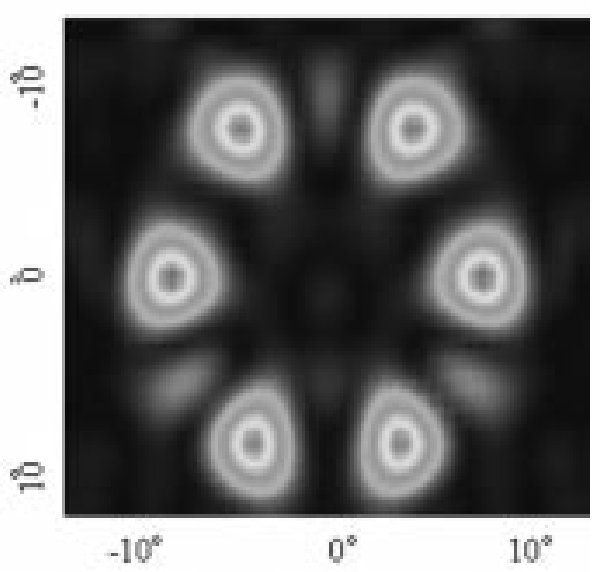

(b)

Fig. 10. The calculated near-field and far-field profiles of a 19-element triangular lattice 2-D array for an: (a) in-phase mode and (b) out-of-phase mode.

nant mode and 2) it has a very small effect on the resonant or nearly-resonant mode which exhibits very little inter-element field.

The effect of enhanced modal discrimination in favor of the resonant modes, by the introduction of $100 \mathrm{~cm}^{-1}$ loss into the inter-element regions, is demonstrated for the $4 \times 4$ antiguided array using the effective index model. The influence of the interelement loss on the total modal loss (i.e., edge radiation loss plus absorption loss) for the in-phase mode $(12,12)$, the lower out-of-phase mode $(9,9)$ and relevant (lowest loss) adjacent modes are shown in Fig. 9. As a result, for $s>2.3 \mu \mathrm{m}$, all modes other than the in-phase mode $(12,12)$ can be suppressed by high radiation loss. Thus, Fig. 9 demonstrates that a $4 \times 4$ array structure designed with an inter-element loss of $100 \mathrm{~cm}^{-1}$ and an inter-element width in the range of $s=2.3-2.8 \mu \mathrm{m}$ is expected to operate in the stable in-phase array mode. The additional loss can be obtained by many ways, such as introducing a highly absorbing layer outside of the cavity region but inside the effective cavity length region, or by reducing the reflectivity of the inter-element region. For example, for our structure (described in Section VI), we can introduce a 7-nm InGaAs $(980 \mathrm{~nm})$ quantum well (QW) inside the thin spacer layers after we grow the first pair of the top DBR. The optical confinement factor of this 7-nm-wide InGaAs QW within the effective cavity length is calculated to be $\Gamma \sim 1 \%$. Assuming a bulk absorption coefficient of $\alpha \sim 10^{4} \mathrm{~cm}^{-1}$, the modal loss due to this absorption layer is about $\Gamma \alpha=100 \mathrm{~cm}^{-1}$. The amount of interelement loss can be adjusted by varying the thickness of the QW (within the critical thickness constraint) as well as its position within the DBR.

The uniform or near-uniform field profile makes the resonant device less sensitive to GSHB and thermal effects, making it suitable for high-power single-mode operation. However, a detailed above-threshold analysis considering mode competition for the 2-D array is still necessary.

\section{TRIANGULAR GEOMETRY 2-D VCSEL ARRAY}

Resonant leaky-wave coupling will occur for other array geometry as long as condition (3) is satisfied. For example, the use of a triangular structure may allow for stronger optical coupling, since there are six nearest neighbors (instead of four neighbors in the previous structure) around each array element. To confirm resonant coupling in a nonrectangular lattice, we studied the resonance behavior of a 19-element triangular geometry array. The calculated in-phase mode near-field and far-field profiles, for two different array element spacings, are shown in Fig. 10(a), indicating that resonant behavior can indeed be achieved for 


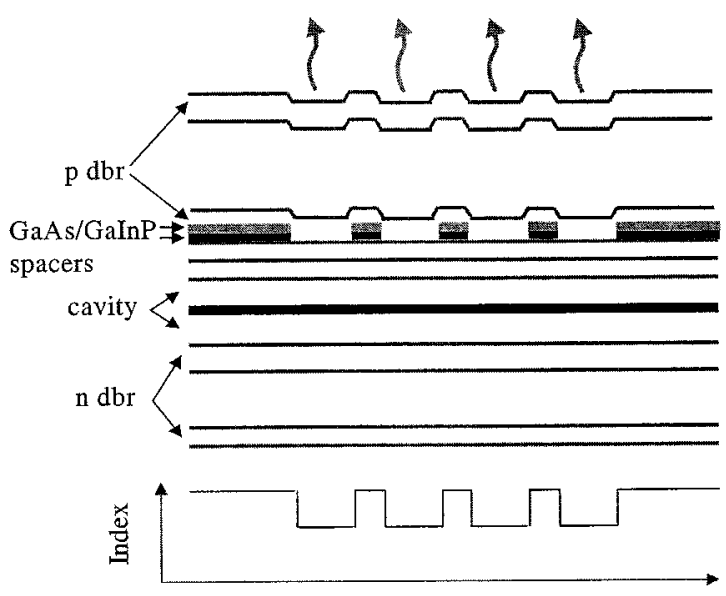

Fig. 11. Schematic cross section of a $4 \times 4$ antiguided VCSEL structure emitting at $980 \mathrm{~nm}$ with element width $d=6 \mu \mathrm{m}: \mathrm{H}^{+}$ion implantation is used for the current confinement to the array.

such a structure. The large radiation losses at resonance, evident from the field profile, are reduced as the number of elements in the array increases. Out-of phase mode behavior [Fig. 10(b)] is more complicated than in the rectangular lattice structures due to the increased number of nearest neighbors. Generally speaking, stronger coupling leads to better modal discrimination in favor of the resonant modes although a comprehensive investigation is still underway.

\section{DEVICE PERFoRMANCE}

\section{A. Structure and Fabrication}

Top-emitting antiguided array structures $(d=6 \mu \mathrm{m}, d n=$ $0.05)$ were fabricated using a two-step MOCVD growth process [20] as shown in Fig. 11. It consists of 32.5 pairs of AlAs-GaAs n-DBR, 23 pairs of $\mathrm{Al}_{0.15}$ GaAs-GaAs p-DBR, and $1-\lambda$ optical cavity that includes three InGaAs QWS GaAs barrier layers, and $\mathrm{Al}_{0.3} \mathrm{GaAs}$ confinement layers for $980-\mathrm{nm}$ emission. The first growth consists of n-DBR, cavity, 1 pair of $\mathrm{p}-\mathrm{DBR}$ and two thin spacer layers of GaAs $(10 \mathrm{~nm})$ and $\mathrm{GaInP}(12 \mathrm{~nm})$. The purpose of these thin layers is to generate an effective index step between the inter-element and element regions. The calculated effective index step for this structure is $\Delta n=0.05$ [20].

We use $\mathrm{H}_{3} \mathrm{PO}_{4}: \mathrm{H}_{2} \mathrm{O}_{2}: \mathrm{H}_{2} \mathrm{O}(1: 1: 5)$ and $\mathrm{HCl}: \mathrm{H}_{2} \mathrm{O}(4: 1)$ to selectively etch away the GaAs and GaInP thin layers under the emitting elements area, and then regrow the remaining top p-DBR. The following processes include defining the current aperture by $\mathrm{H}^{+}$ion implantation, and making top and bottom ohmic contacts as well as opening the optical window. The interelement (high-index region) width $s$ was adjusted to be between $2-3.5 \mu \mathrm{m}$ to select either the in-phase or out-of-phase resonance condition. Note that in these initial structures we do not intentionally include absorbing layers for inter-element loss to aid the suppression of nonresonant modes, as discussed above.

\section{B. Spectrum and Far Field}

CW single-mode in-phase emission is observed from arrays with inter-element widths of $s=2.5 \mu \mathrm{m}$ with the characteristics of an on-axis-lobe far-field pattern. At $s=3.0 \mu \mathrm{m}$, single out-of-phase mode operation with the resulting four-lobe

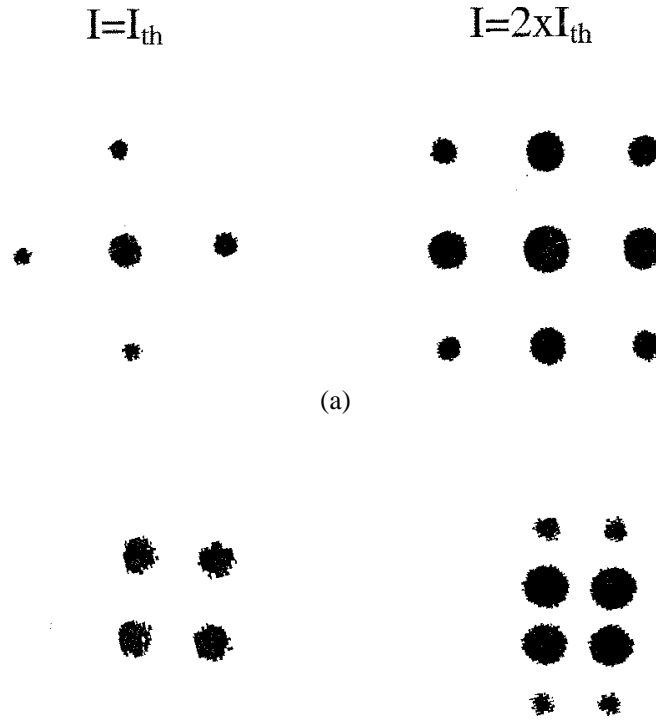

$12^{\circ}$

(b)

Fig. 12. Measured CW far-field patterns for a $4 \times 4$ array operating in the: (a) in-phase mode $(s=2.5 \mu \mathrm{m})$ and (b) out-of-phase $(s=3.0 \mu \mathrm{m})$ mode.

far-field pattern is obtained. The measured $\mathrm{CW}$ far-field emission pattern for an array with $s=2.5 \mu \mathrm{m}$ (in-phase) and $s=$ $3.0 \mu \mathrm{m}$ (out-of-phase), as captured by a CCD camera, are shown in Fig. 12. As the driving current increases, the minor side-lobes become viewable, in excellent agreement with the calculated far-field shown in Fig. 5. The angular lobe separation and widths are obtained from the 1-D scan (Fig. 13) of the far-field intensity for the arrays with a full width half maximum (FWHM) for the in-phase mode of about $2^{\circ}$, and a side lobe separation of $13.5^{\circ}$, in agreement with the calculation. We have also fabricated $2 \times 2$ and $3 \times 3$ arrays, operating in-phase, and the measured far-field FWHM of the central lobe is shown in Fig. 14 as a function of the number of array elements, $N$ (for a $N \times N$ array), showing excellent agreement with theory. The emission patterns remain stable up to the maximum thermally limited $\mathrm{CW}$ output power ( 1-2 $\mathrm{mW}$ ), and pulsed output power greater than $10 \mathrm{~mW}$. The measured CW spectrum is shown in Fig. 15, indicating all elements remain phase locked at the same frequency over the $\mathrm{CW}$ operating range.

Higher CW output powers are mainly limited due to the unoptimized top DBRs and intensive heating for such closely packed arrays. Nevertheless, our results are in general agreement with the calculation shown in Fig. 6, provided that the modal loss curves are shifted toward either higher or lower $s$ values by approximately $0.8 \mu \mathrm{m}\left(\lambda_{1} / 2\right)$. For this case, the in-phase nonresonant mode will have lowest loss at $s=2.5 \mu \mathrm{m}$. This shift can occur due to a smaller index step ( $\Delta n=0.036)$ than the design target of $\Delta n=0.05$. Since the fabricated structure does not contain inter-element loss, the lasing modes observed correspond to either the nonresonant in-phase (for $s=2.5 \mu \mathrm{m}$ ) or the nonresonant out-of-phase (for $s=3.0 \mu \mathrm{m}$ ). Recently, we have successfully implemented 


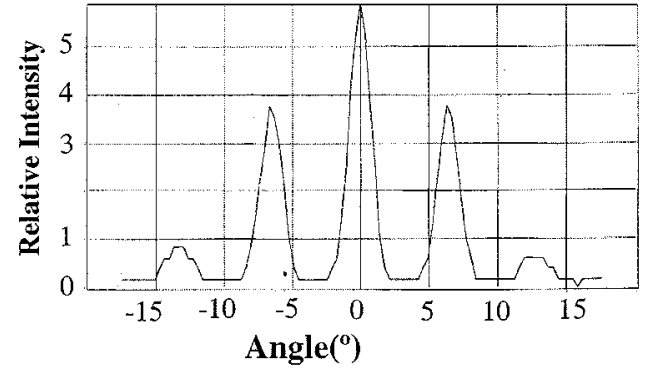

(a)

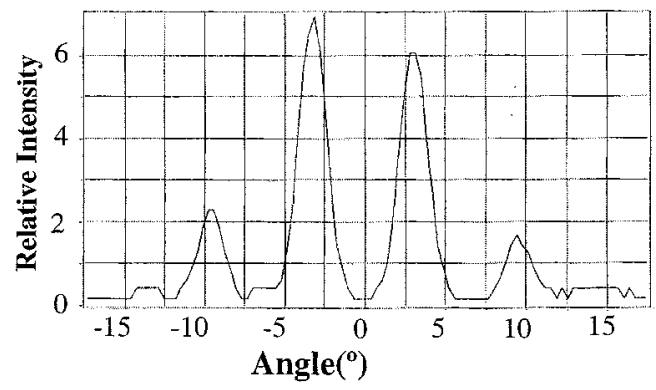

(b)

Fig. 13. 1-D scan of the measured far-field patterns in Fig. 12: (a) in-phase mode and (b) out-of-phase mode.

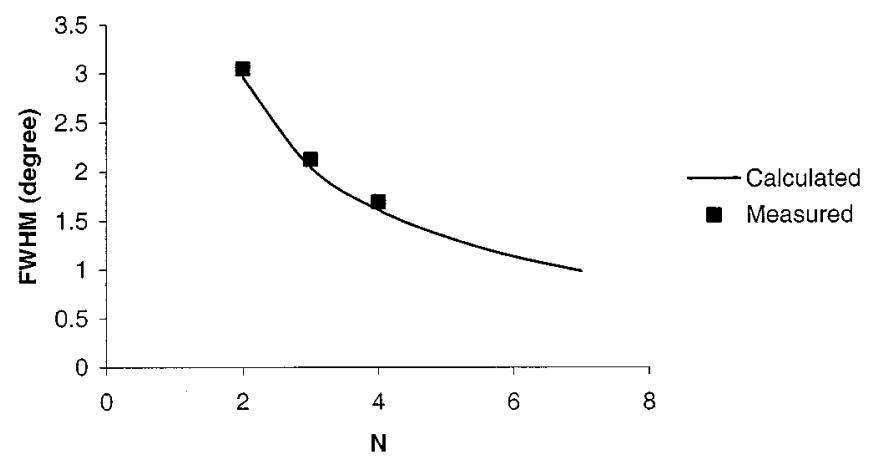

Fig. 14. Comparison between theory and measured FWHM of the central far-field lobe for $2 \times 2,3 \times 3$, and $4 \times 4$ arrays operating in the in-phase mode.

inter-element loss into the above-described nonresonant array structures, thereby achieving the first observation of resonant in-phase emission [35]. Although these $4 \times 4$ arrays (threshold currents are about $25 \mathrm{~mA}$ with a $40 \times 40 \mu \mathrm{m}^{2}$ pumping area) exhibit maximum $\mathrm{CW}$ power limited to $2 \mathrm{~mW}$ due to thermal effects and misalignment between the gain and optical mode, they exhibit high peak-pulsed power of over $40 \mathrm{~mW}$. Future work will be focused on how to effectively minimize heating and include the inter-element loss to select the resonant or near-resonant in-phase mode.

\section{Thermal Effects on Array Performance}

For the densely packed top-emitting array (can be approximated as a large modal area device) with a thick substrate, as shown in Fig. 11, the output is mainly thermally limited under higher driving currents due to the lowered differential quantum

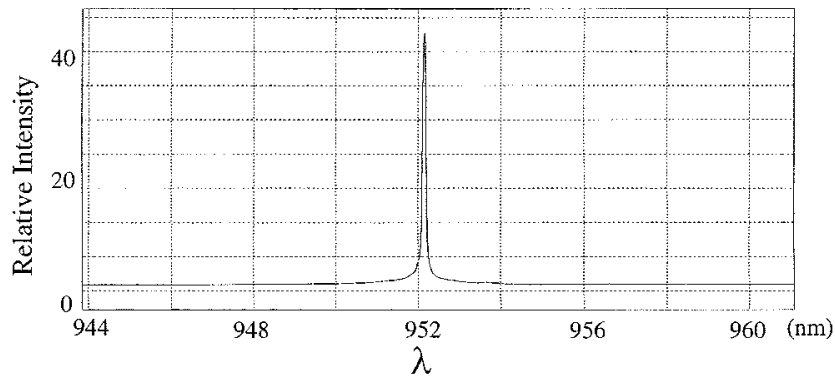

(a)

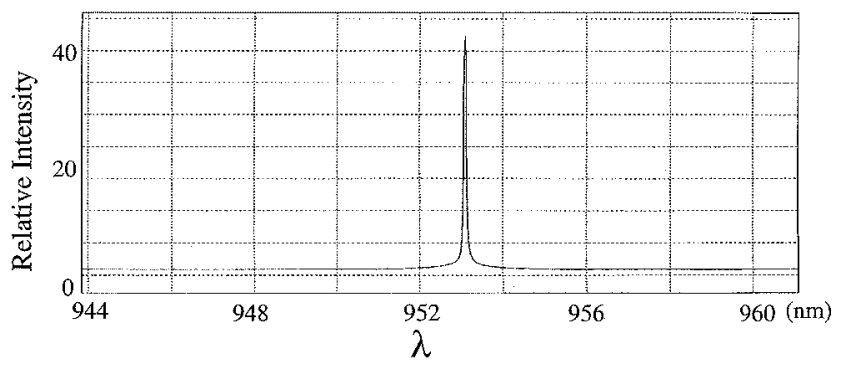

(b)

Fig. 15. Measured CW spectrum at (a) $I_{\mathrm{th}}$ and (b) $2 \times I_{\mathrm{th}}$ for the in-phase mode $(s=2.5 \mu \mathrm{m})$.

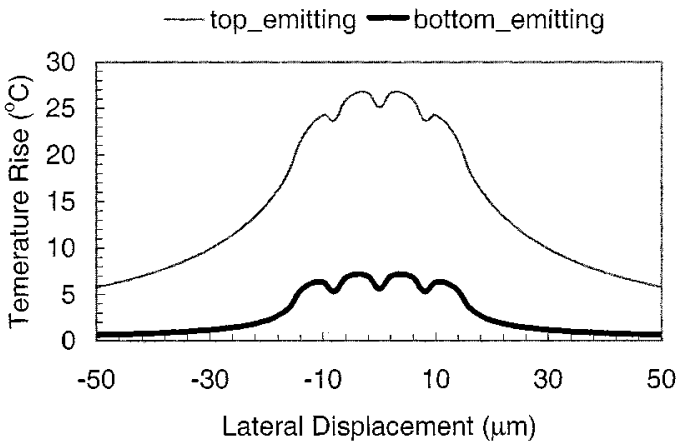

Fig. 16. Calculated temperature distribution across a $4 \times 4$ VCSEL array assuming 5-mW power dissipation per VCSEL element.

efficiency. The thermal impedance of such structure can be expressed as

$$
Z_{T}=(2 \xi D)^{-1}
$$

where $\xi$ is the average material thermal conductivity between the active region and heat sink, $D$ is the whole width of the 2-D array [36]. The temperature increase under driving current $I$ is

$$
\Delta T=Z_{T} P_{D}
$$

where $P_{D}$ is the dissipated power inside the device: $P_{D}=$ $P_{\text {in }}(1-\eta)$ in which $\eta$ is the wall-plug efficiency and $P_{\text {in }}$ is the input power

$$
P_{\text {in }}=I^{2} R_{s}+I\left(V_{s}+V_{d}\right)
$$

where $R_{s}$ is the series resistance, $V_{s}$ is the current-independent series voltage, and $V_{d}$ is the ideal diode voltage.

For a rough approximation, assuming that the current is injected uniformly from the p-side (no current crowding effect for 

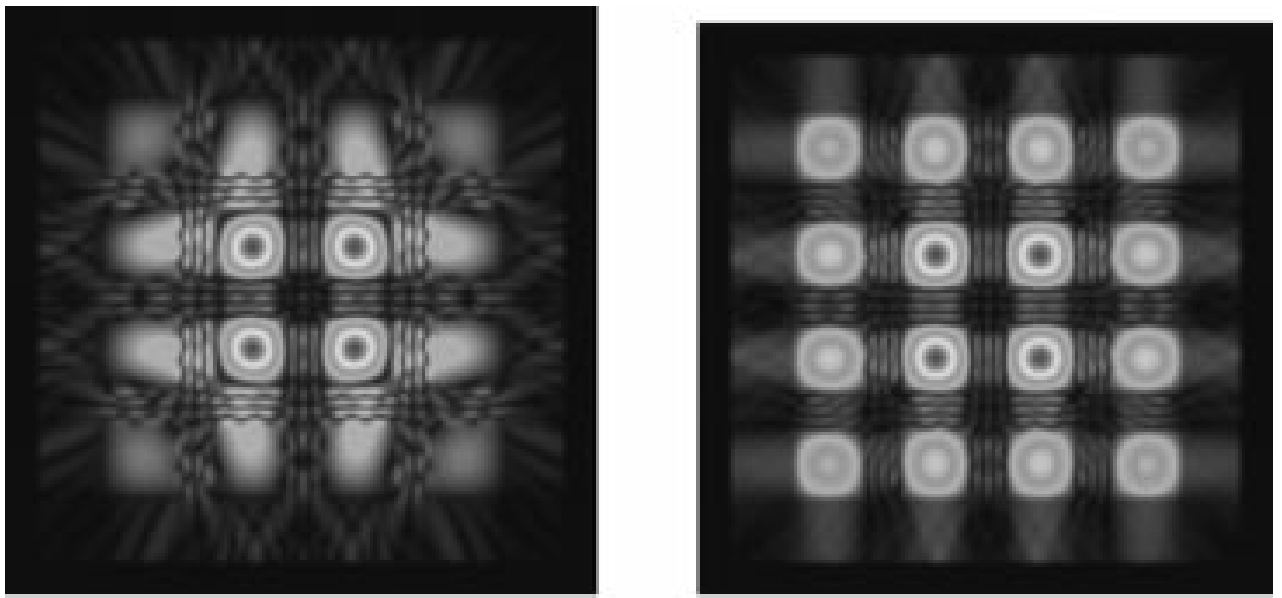

(a)

$8 \mu \mathrm{m}$

(b)

Fig. 17. Computed field profiles corresponding to the resonant in-phase mode including the thermally induced index profile for: (a) junction-up and (b) junction-down configuration. Strong self-focusing occurs for the junction-up geometry.

a large array), $I$ is proportional to the square of $D$. Thus, from (15)-(17), $\Delta T$ is roughly proportional to $D$. Moreover, thermal cross talk for such closely packed phase-locked arrays will raise the temperature even further [37]. These increased temperatures will inevitably modify the device waveguiding characteristics and resulting beam shape, and reduce the differential quantum efficiency and output power. Because the heat is primarily generated in the cavity and thin top p-DBR regions, mounting the p-side down on an effective heat sink will be beneficial, which has been proven in the large-aperture VCSEL and large spatially-incoherent 2-D VCSEL arrays [24].

A 3-D finite element analysis is employed to study the thermal behavior of a typical $4 \times 4$ array for both of the top-emitting and bottom-emitting cases, and the resulting 1-D cut of the cavity region's temperature increase is plotted in Fig. 16. These calculations assume a copper heatsink held at $25{ }^{\circ} \mathrm{C}$ for both junction-up and junction-down configurations. We use this data to modulate the thermally adjusted array built-in refractive index profile, assuming that the temperature coefficients of the refractive index for GaAs and AlAs are $\beta=4 \times 10^{-4} \mathrm{~K}^{-1}$ [38] and $1 \times 10^{-4} \mathrm{~K}^{-1}$ [39], [40], respectively. Fig. 17 shows that, for a resonant mode with a uniform near-field, as shown in Fig. 4 for $s=2.4 \mu \mathrm{m}$, the thermal effect is different for the top-emitting and bottom-emitting designs. While the modal intensity of the top-emitting array is severely focused into a cosine shape profile, the bottom-emitting array's modal profile is much less affected. We also observe that the modal radiation loss for the top-emitting and bottom-emitting cases are increased and reduced, respectively. Therefore, bottom-emitting array implementation is essential for high-power, coherent 2-D VCSEL array operation. Preliminary results from junction-down devices demonstrate $10 \mathrm{~mW} \mathrm{CW}$ coherent emission, a significant improvement from the junction-up structures. Further improvement is expected through the use of high thermal conductivity diamond heat-spreaders and further device optimization.

\section{CONCLUSION}

2-D antiguided VCSEL arrays support a multitude of leaky modes, which are highly dependent on the array geometry. When the inter-element spacing is equal to an integer number times of the half lateral wavelength, the array is under a resonance condition with its resonant in-phase or out-of-phase modes having uniform near-field and negligible inter-element field, which is less vulnerable to GSHB and thermal effects. The radiation loss of the resonant mode is found to be inversely proportional to the array number $N$ with $\alpha_{N \times N}=\alpha_{1 \times 1} / N$.

Intermediate-order or adjacent modes are quite complicated due to the 2-D array geometry. Most of theses modes have higher edge radiation loss than the in-phase or out-of-phase mode, except for those exhibiting a resonant or nonresonant behavior in at least one direction of the array. Nevertheless, they all have significant inter-element fields, allowing inter-element losses to be used to suppress lasing.

Due to the small variance of the transverse relative optical confinement factor, the 3-D gain overlap is not a significant factor in mode selection. However, inter-element loss can suppress the nonresonant modes effectively and make the resonant or near-resonant in-phase mode favored to lase. Other 2-D array geometries, such as a triangular lattice, also exhibits resonant behavior, provided that the inter-element spacing satisfies the 1-D resonant condition.

Fabricated $N \times N(N=2,3,4)$ antiguided VCSEL arrays show that, with suitable inter-element spacing and index step, the array can select either the in-phase or out-phase mode. The measured beam profiles for both the in-phase and out-of-phase modes are diffraction-limited, indicating single-array mode operation. Due to the inherent heating problem of the large-area VCSEL device, the output power is quite limited for the large top-emitting arrays (1-2 mW CW and $40 \mathrm{~mW}$ pulsed). From a thermal analysis, we have shown that the top-emitting structure will result in strong self-focusing due to thermal lensing. By comparison, a bottom-emitting structure is found to be more suitable for coherent high-output-power emission. 


\section{REFERENCES}

[1] C. Jung, R. Jager, M. Grabherr, P. Schnitzer, R. Michalzik, B. Weigl, S. Muller, and K. J. Ebeling, " $4.8 \mathrm{~mW}$ single mode oxide confined topsurface emitting vertical-cavity laser diodes," Electron Lett., vol. 33, pp. $1790-1791,1997$.

[2] S. W. Z. Mahmoud, U. J. Unold, W. Schmid, R. Jager, R. Michalzik, and K. J. Ebeling, "Analysis of longitudinal mode wave guiding in verticalcavity surface-emitting lasers with long monolithic cavity," Appl. Phys. Lett., vol. 78, pp. 586-588, 2001.

[3] J. P. Van Der Ziel, D. G. Deppe, N. Chand, G. J. Zydzik, and S. N. G. Chu, "Characteristics of single- and two-dimensional phase couples arrays of vertical cavity surface emitting GaAs-AlGaAs lasers," IEEE J. Quantum Electron., vol. 26, pp. 1873-1881, 1990.

[4] M. Orenstein, E. Kapon, N. G. Stoffel, J. P. Harbison, L. T. Florez, and J. Wullert, "Two-dimensional phase-locked arrays of vertical-cavity semiconductor lasers by mirror reflectivity modulation," Appl. Phys. Lett., vol. 58, pp. 804-806, 1991.

[5] R. A. Morgan, K. Kojima, T. Mullally, G. D. Guth, M. W. Focht, R. E. Leibenguth, and M. Asom, "High-power coherently coupled $8 \times 8$ vertical cavity surface emitting laser array," Appl. Phys. Lett., vol. 61, pp. 1160-1162, 1992.

[6] P. L. Gourley, M. E. Warren, G. R. Hadley, G. A. Vawter, T. M. Brennan, and B. E. Hammons, "Coherent beams from high efficiency two-dimensional surface-emitting semiconductor laser arrays," Appl. Phys. Lett., vol. 58 , pp. 890-892, 1991.

[7] D. Scifres, W. Streifer, and R. D. Burnham, "Experimental and analytic studies of coupled multiple stripe diode lasers," IEEE J. Quantum Electron., vol. QE-15, pp. 917-922, 1979.

[8] J. K. Butler, D. E. Ackley, and D. Botez, "Coupled-mode analysis of phase-locked injection laser arrays," Appl. Phys. Lett., vol. 44, pp. 293-295, 1984

[9] E. Kapon, J. Katz, and A. Yariv, "Supermode analysis of phase-locked semiconductor laser arrays," Opt. Lett., vol. 9, pp. 125-127, 1984.

[10] M. E. Warren, P. L. Gourley, G. R. Hadley, G. A. Vawter, T. M. Brennan, B. E. Hammons, and K. L. Lear, "On-axis far-field emission from two-dimensional phase-locked vertical cavity surface-emitting laser arrays with an integrated phase-corrector," Appl. Phys. Lett., vol. 61, pp. 1484-1486, 1992.

[11] K.-L. Chen and S. Wang, "Spatial hole burning problems in evanescently coupled semiconductor laser arrays," Appl. Phys. Lett., vol. 47, pp. 555-557, 1985

[12] R. A. Morgan and K. Kojima, "Optical characteristics of two-dimensional coherently coupled vertical cavity surface emitting laser arrays," Opt. Lett., vol. 18, pp. 352-354, 1993.

[13] D. E. Ackley and W. H. Engelmann, "High-power leaky-mode multiplestripe laser," Appl. Phys. Lett., vol. 39, pp. 27-29, 1981.

[14] D. Botez, M. Jansen, L. J. Mawst, G. Peterson, and T. J. Roth, "Wattrange, coherent, uniphase powers from phase-locked arrays of antiguided diode lasers," Appl. Phys. Lett., vol. 58, pp. 2070-2072, 1991.

[15] Y. A. Wu, G. S. Li, R. F. Nabiev, K. D. Choquette, C. Caneau, and C. J. Chang-Haisnain, "Single-mode, passive antiguide vertical cavity surface emitting laser," IEEE J. Select. Topics Quantum Electron., vol. 1, pp. 629-637, 1995

[16] T. H. Oh, M. R. McDaniel, D. L. Huffaker, and D. G. Deppe, "Cavity-induced antiguiding in a selectively oxidized vertical-cavity surface-emitting laser," IEEE Photon. Technol. Lett., vol. 10, pp. 12-14, 1998.

[17] K. D. Choquette, G. R. Hadley, H. Q. Hou, K. M. Geib, and B. E. Hammons, "Leaky mode vertical cavity lasers using cavity resonance modification," Electron Lett., vol. 34, pp. 991-992, 1998.

[18] D. K. Serkland, K. D. Choquette, G. R. Hadley, K. M. Geib, and A. A. Allerman, "Two-element phased array of antiguided vertical-cavity lasers," Appl. Phys. Lett., vol. 75, pp. 3754-3756, 1999.

[19] D. Zhou and L. J. Mawst, "Two-dimensional phase-locked antiguided vertical-cavity surface-emitting laser arrays," Appl. Phys. Lett., vol. 77 , pp. 2307-2309, 2000.

[20] D. Zhou and L. J. Mawst, "Simplified-antiresonant reflecting optical waveguide-type vertical-cavity surface-emitting lasers," Appl. Phys. Lett., vol. 76, pp. 1659-1671, 2000.

[21] D. Botez, L. J. Mawst, and G. Peterson, "Resonant leaky-wave coupling in linear arrays of antiguides," Electron Lett., vol. 24, pp. 1328-1330, 1988.

[22] D. Botez, L. J. Mawst, G. Peterson, and T. J. Roth, "Resonant optical transmission and coupling in phase-locked diode-laser arrays of antiguides: The resonant-optical-waveguide array," Appl. Phys. Lett., vol. 54, pp. 2183-2185, 1989.
[23] G. R. Hadley, "Index-guided arrays with large index step," Opt. Lett., vol. 14 , pp. 308-310, 1989

[24] M. Grabherr, M. Miller, R. Jager, R. Michalzik, U. Martin, H. J. Unold, and K. J. Ebeling, "High-power VCSEL's: Single devices and densely packed 2-D-arrays," IEEE J. Select. Topics Quantum Electron., vol. 5, pp. 495-502, 1999

[25] G. R. Hadley, "Effective index model for vertical-cavity surface-emitting lasers," Opt. Lett., vol. 20, pp. 1483-1485, 1995.

[26] D. Botez, L. J. Mawst, G. L. Peterson, and T. J. Roth, "Phase-locked arrays of antiguidess: Modal content and discrimination," IEEE J. Quantum Electron., vol. 26, pp. 482-495, 1990.

[27] P. Yeh, Optical Waves in Layered Media. New York: Wiley, 1988, pp. $87-88$.

[28] R. F. Nabiev and D. Botez, "Comprehensive above-threshold analysis of antiguided diode laser arrays," IEEE J. Select. Topics Quantum Electron., vol. 1, pp. 138-149, 1995.

[29] D. Botez, P. Hayashida, L. J. Mawst, T. J. Roth, and G. Peterson, "Diffraction-limited in-phase-mode operation from uniform arrays of antiguides with enhanced interelement loss," Electron Lett., vol. 25, pp. $1282-1284,1989$.

[30] G. R. Hadley, "Two-dimensional waveguide modeling of leaky-mode arrays," Opt. Lett., vol. 14, pp. 859-861, 1989.

[31] L. J. Mawst, D. Botez, T. R. Roth, W. W. Simmons, G. Peterson, M. Jansen, J. Z. Wilcox, and J. J. Yang, "Phase-locked array of antiguided lasers with monolithic spatial filter," Electron Lett., vol. 25, pp. 365-366, 1989.

[32] J. Z. Wilcox, W. W. Simmons, D. Botez, M. Jansen, L. J. Mawst, G. Peterson, T. J. Wilcox, and J. J. Yang, "Design considerations for diffraction coupled arrays with monolithically integrated self-imaging cavities," Appl. Phys. Lett., vol. 54, pp. 1848-1850, 1989.

[33] R. W. Engelmann and D. Kerps, "Leaky modes in active three-layer slab waveguides," Proc. Inst. Elect. Eng., pt. I, vol. 127, pp. 330-336, 1980.

[34] S. W. Corzine, R. S. Geels, J. W. Scott, R. Yan, and L. A. Coldren, "Design of Fabry-Perot surface-emitting lasers with a periodic gain structure," IEEE J. Quantum Electron., vol. 25, pp. 1513-1524, 1989.

[35] D. Zhou, A. Napartovich, N. N. Elkin, D. V. Vysotsky, and L. J. Mawst, "Modal characteristics of 2-D antiguided VCSEL arrays," in Proc. Photonic West 2002, Vertical Cavity Surface Emitting Lasers VI (Conf. 4649), vol. 35, San Jose, CA, Jan. 2002, pp. 168-178.

[36] L. A. Coldren and S. W. Corzine, Diode Lasers and Photonic Integrated Circuits. New York: Wiley, 1995, p. 56.

[37] M. Osinski and W. Nakwaski, "Thermal analysis of closely-packed twodimensional etched-well surface-emitting laser arrays," IEEE J. Select. Topics Quantum Electron., vol. 1, pp. 681-696, 1995.

[38] H. C. Casey and M. B. Panish, Heterostructure Lasers. New York: Academic, 1978, pt. A, p. 31

[39] H. G. Grimmeiss and B. Monemar, "Temperature dependence of the refractive index of AlAs and AlP," Phys. Stat. Sol., (a), vol. 5, pp. 109-114, 1971.

[40] B. Jensen and W. D. Jensen, "The refractive index near the fundamental absorption edge in $\mathrm{Al}_{x} \mathrm{Ga}_{1-x}$ As tenary compound semiconductors," IEEE J. Quantum Electron., vol. 27, pp. 40-45, 1991.

[41] J. K. Butler, D. E. Ackley, and D. Botez, "Coupled-mode analysis of phase-locked injection laser arrays," Appl. Phys. Lett., vol. 44, p. 935, 1984.

[42] E. Kapon, J. Katz, and A. Yariv, "Supermode analysis of phase-locked semiconductor laser arrays," Opt. Lett., vol. 9, p. 318, 1984.

Delai Zhou (M'01) was born in Shenyang, China, in 1975. He received the B.S degree in nuclear physics from Peking University, Beijing, China, in 1995 and the M.S.E.E. degree in 1998 from the University of Wisconsin-Madison, where he is currently working toward the Ph.D. degree in the area of photonics. His dissertation research focuses on the development of high-power single-mode VCSELs and 2-D phase-locked VCSEL arrays utilizing the antiguided structures.

In 1996, he joined the Department of Electrical and Computer Engineering of the University of Wisconsin-Madison, where he is currently a Research Assistant in the Reed Center for Photonics. 
L. J. Mawst (M'88-SM'93) was born in Chicago, IL, in 1959. He received the B.S. degree in engineering physics and the M.S. and Ph.D. degrees in electrical engineering from the University of Illinois at Urbana-Champaign in 1982, 1984, and 1987, respectively. His dissertation research involved the development of index-guided semiconductor lasers and laser arrays grown by MOCVD.

He joined TRW, Inc., Redondo Beach, CA, in 1987, where he was a Senior Scientist in the research center, engaged in design and development of semiconductor lasers using MOCVD crystal growth. He is co-inventor of the Resonant Optical Waveguide (ROW) antiguided array and has contributed to its development as a practical source of high coherent power. He developed a novel single-laser structure, the ARROW laser, as a source for coupling high powers into fibers. He has also been involved in the development of 2-D coherent surface-emitting arrays, vertical-cavity surface emitters, and distributed-feedback laser structures. He is currently an Associate Professor at the University of Wisconsin-Madison, where he is involved in the development of novel III/V compound semiconductor device structures, including vertical cavity surface emitters (VCSELs), active photonic lattice structures, and high-power Al-free diode lasers. He has authored or co-authored more than 100 technical papers and holds 13 patents.

Dr. Mawst received the TRW Group Level Chairman's Award for his work on the ROW antiguided array.
Zheng Dai (M'01) was born in Liaoning, China, in 1963. He received the B.S. degree in applied physics from Hefei University of Technology, Hefei, China, in 1985, the M.S. degree in optics from the Chinese Academy of Science in 1990, and the Dr.-Ing. degree in optoelectronics from University of Ulm, Ulm, Germany, in 1997.

During 1998 and 2000, he was a R\&D Scientist with JDS Uniphase Corporation. In 2000, he joined CoreTek Inc. (later acquired by Nortel Networks Corporation) as a Senior Engineer. Recently, he became a Project Manager at Alfalight Inc., Madison, WI. His research interests cover various diode lasers and DWDM components, in particular, developing these advanced photonic components into industrial products. He was a major contributor in developing the products of a 40-channel diffraction grating DWDM DEMUX, C-band/L-band wavelength locker, MEM tunable filter, MEM-tunable VCSELs, and broad-area high-power diode lasers. 